\title{
Developing an empirically grounded framework to assess interdisciplinarity of student explanations of everyday phenomena
}

\author{
K. K. Mashood ${ }^{1,2}$, Vashti Sawtelle,3, Charles W. Anderson ${ }^{4}$, Rebecca L. Matz ${ }^{2}$, Emily \\ E. Scott ${ }^{2,4}$, and Sonia M. Underwood ${ }^{5,6}$ \\ ${ }^{I}$ Department of Physics and Astronomy, Michigan State University, East Lansing, MI 48824 \\ ${ }^{2}$ CREATE for STEM Institute, Michigan State University, East Lansing, MI 48824 \\ ${ }^{3}$ Lyman Briggs College, Michigan State University, East Lansing, MI 48824 \\ ${ }^{4}$ Department of Teacher Education, Michigan State University, East Lansing, MI 4882 \\ ${ }^{5}$ Department of Chemistry and Biochemistry, Florida International University, Miami, FL 33199 \\ ${ }^{6}$ STEM Transformation Institute Florida International University, Miami, FL 33199
}

Interdisciplinary thinking and reconciliation is integral to developing a coherent understanding of science. Projects like NEXUS are significant advancements in this regard and have created a need for new assessment tools. This paper discusses the development of an empirically grounded framework to assess the interdisciplinarity of students' explanations of everyday phenomena. As a preliminary analysis, we examine interview transcripts from two undergraduate students explaining the solidification of liquid egg white upon boiling. The extent to which students invoke different disciplines is considered as a criterion to assess the interdisciplinarity of their explanations. We carry this out by mapping the vocabulary and knowledge elements involved in student explanations to content presentations in introductory level physics, chemistry and biology textbooks.

\section{INTRODUCTION AND MOTIVATION}

The academic community is increasingly recognizing the significance of interdisciplinary thinking and education $[1,2]$. Perhaps the complex problems of our times and major trends at research frontiers have brought us to a point where the traditional notions of isolated disciplines have started appearing naive and archaic. We require the next generation not to be "students of some subject matter, but students of problems; and problems may cut right across the borders of any subject matter or discipline" $[1, p .16]$. For this to happen, our classroom practices need to be transformed accordingly. Connecting and integrating knowledge from across disciplines can also foster depth and coherence in student thinking and learning. It is particularly true in case of those concepts (like energy) which are complex and have multiple connotations associated with them. This varied descriptions depending on the context makes reconciliation between them, a difficult task for students.

A look into current undergraduate practices in the natural sciences (physics, chemistry and biology) reveals that the students mostly stay confined to a single discipline. To combat this narrow focus, we need to provide them with opportunities in interdisciplinary settings where they learn to invoke and integrate knowledge from multiple disciplines effectively. Perhaps no other context is as inclusive, relevant and authentic to students' experiences as everyday phenomena. However, their inherent interdisciplinary nature possibly stymies their full-fledged entry into classroom discourses. The traditional science courses, confined within the framework of single disciplines, are ill-suited for any serious discussion of everyday phenomena. With increasing emphasis on interdisciplinarity, we are in a position to harness the underexplored pedagogic potential of these phenomena. This can inform and supplement interdisciplinary initiatives like the introductory physics for life sciences (IPLS) courses [3].

In this paper we discuss the preliminary stages of developing an empirically grounded framework for assessing the interdisciplinarity of student explanations for a set of everyday phenomena. We focus on how students invoke and integrate knowledge from physics, chemistry and biology to construct their explanation. The phenomena, rationale behind their choice and details of data collection are discussed in the next section. This is followed by a discussion of preliminary data analysis in Section III. Our discussion ends with the concluding remarks in Section IV.

\section{METHODOLOGY AND DATA COLLECTION}

We chose a set of five everyday phenomena as contexts to examine student reasoning across the disciplines. Framed in the form of questions they are: 1) What happens to egg white when an egg is boiled? 2) How can someone sneezing make another person sick? 3) How does an ultrasound machine produce the image of a gallstone? 4) How do blisters form upon touching a hot pan? 5) How do Hollywood actors build huge muscles? The phenomena were chosen such that they are familiar to students and require an optimal amount of content to provide a 
satisfactory explanation. It was also required that scientific explanations of these phenomena would reflect "threedimensional" learning objectives, [4] namely that they involve core ideas from physics, chemistry and biology (as confirmed by faculty), scientific practices, and crosscutting concepts. For example, from the researchers' perspective a satisfactory explanation of the solidification of egg white would include mechanism of heat transfer (physics), energy and bond breaking (chemistry) and protein denaturation (biology), among other ideas.

Identification of the phenomena was followed by conducting semi-structured student interviews. Twelve undergraduate life science majors who were currently enrolled in an algebra based physics course and had previously taken a course in chemistry were interviewed. Each interview lasted about an hour in which 3-4 phenomena were covered. Students were asked to explain each phenomenon on the basis of what they had learned in different science courses as well as their real world experiences. As part of the scaffolding for the interviews, student reasoning was probed at different scales in that if their explanations were mainly macroscopic, students were prompted to reason at the molecular level. Ten minutes at the end of each interview were devoted for meta-level conversations regarding the disciplines students invoked in their explanations, extent of connections they made between disciplines and their personal experiences. The interviews were video recorded and the audio was transcribed verbatim for analysis.

Based on insights from the interviews, we developed a set of assessment questions as an open-ended activity and administered it to over 1000 students on an online platform called beSocratic, a system that allows for free-form drawn and written responses [5]. For the purposes of this paper, we will focus on the assessment scheme developed from looking at the interview data.

\section{PRELIMINARY DATA ANALYSIS OF INTERVIEWS}

Explaining everyday phenomena with scientific concepts gave students an opportunity to invoke and integrate what they have learned in different science disciplines. However, the data analysis reveals that student explanations vary greatly in both of these dimensions. In this section we focus on how to assess the extent to which students invoke various disciplines in their explanation.

\section{A. Using student reflections to identify disciplinary ideas}

We directly asked the students at the end of the interviews as well as in the written task. The excerpts below from the semi-structured interviews are illustrative examples.
I: [In regards to the egg phenomenon] When you were thinking about these questions, were you drawing on your experience from different disciplines at all or one really resonated with you?

S1: Yeah. So the egg was chemistry and a little bit of biology. This one was my marine biology class from the zoology department. Not marine biology, oceanography.

S2: That was probably for me more about biology and chemistry related. A physics person may be able to describe it in physics terms but for me probably more biology.

Student responses were broad with regard to tracing the disciplinary roots of their ideas. Like the examples above, their responses were often limited to the mention of the disciplines and the corresponding courses from which they think they drew most.

\section{B. Textbook analysis to determine disciplinary ideas}

To get a more detailed and objective picture we developed a coding scheme operationalizing the disciplines. We identified the vocabulary and knowledge elements involved in student explanations and mapped them to content presentations in introductory level physics, chemistry and biology textbooks (see Table I). Knowledge elements are essentially low-inference descriptions of student responses. Vocabulary, as listed in Table I, underscores the focus of our analysis in the corresponding student response. In conjunction with textbook indices they directed the process of mapping of knowledge elements. For example, consider Natalie's response in Table I. We searched the indices of textbooks to find pages where discussions involving the listed vocabularies (say entropy) appear. At this stage one popular textbook from each discipline is being analyzed [68]. The proximity of the knowledge elements to presentation in a particular disciplinary textbook is considered to be a measure of the invoked idea belonging to that discipline. For example, consider the excerpt from Natalie's explanation of the phenomenon of solidification of egg white on boiling, in Table I.

Natalie is trying to explain the phenomenon in terms of entropy. She argues that a change from the liquid to solid state decreases the entropy because solids are rigid and cannot be easily rearranged in shape or volume like a liquid. It is plausible that she considers this rigidity akin to more order or decreased disorder, which in turn is perceived as a decrease in entropy. Textbook analysis reveals that entropy is treated in detail by physics and chemistry. However, chemistry focuses on thermodynamics (entropy included) in the perspective of phase change as an explicit subtopic. In contrast, the physics textbook subsumes this discussion under two worked out problems calculating entropy change 
TABLE I: Textbook analysis to assess the extent to which students invoke different disciplines in their explanation of what happens to an egg white when boiled. Vocabulary and knowledge elements contained in the student response are identified and mapped to presentations in chemistry, physics and biology textbooks.

\begin{tabular}{|c|c|c|c|}
\hline Student response & Vocabulary & Knowledge elements & Textbook quote \\
\hline $\begin{array}{l}\text { Natalie: You're } \\
\text { decreasing the entropy } \\
\text { of it because you're } \\
\text { forming it into a solid, } \\
\text { you're changing its } \\
\text { state, less } \\
\text { arrangements, less } \\
\text { movement because it's } \\
\text { not a liquid anymore. }\end{array}$ & $\begin{array}{l}\text { entropy, } \\
\text { change of } \\
\text { state, solid, } \\
\text { liquid }\end{array}$ & $\begin{array}{l}\text { Entropy decreases } \\
\text { because the egg white } \\
\text { becomes solid. } \\
\text { There is a change of } \\
\text { state from liquid to } \\
\text { solid. } \\
\text { There are less } \\
\text { arrangements, less } \\
\text { movements in solids } \\
\text { than liquids. }\end{array}$ & $\begin{array}{l}\text { Chemistry: In summary, } \\
\text { we generally expect the } \\
\text { entropy of a system to } \\
\text { increase for processes in } \\
\text { which ...Liquids or } \\
\text { solutions form from } \\
\text { solids [7, p. } 798] \\
\text { Physics: ...How much } \\
\text { does the entropy of the } \\
\text { water-ice system change } \\
\text { during the freezing } \\
\text { process? [6,p.639] }\end{array}$ \\
\hline $\begin{array}{l}\text { Julie: So it's more like } \\
\text { the energy transfer } \\
\text { from the heat goes to } \\
\text { the water and the } \\
\text { water energy transfers } \\
\text { to the egg... So } \\
\text { basically you can't } \\
\text { destroy (unclear), you } \\
\text { just transfer around. }\end{array}$ & $\begin{array}{l}\text { energy, } \\
\text { heat, } \\
\text { transfer }\end{array}$ & $\begin{array}{l}\text { Energy from heat } \\
\text { goes to water. } \\
\text { Energy in water } \\
\text { transfers to the egg. }\end{array}$ & 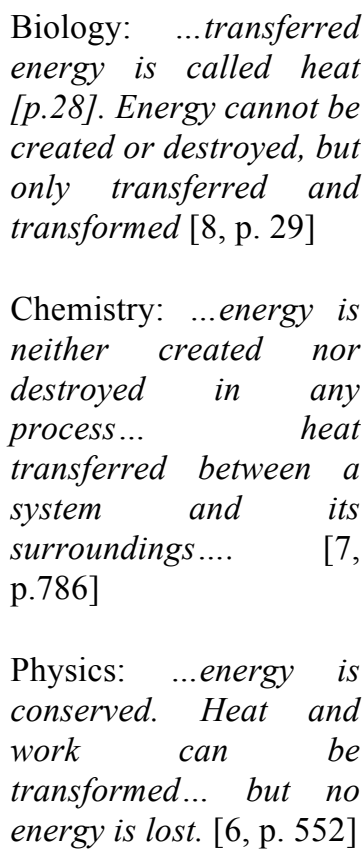 \\
\hline
\end{tabular}

associated with warming and freezing of water. The description of entropy in the biology text is very brief, restricted to a couple of sentences. Therefore, on the basis of our textbook analysis, Natalie's response in Table I is coded as strongly representative of chemistry and moderately of physics. 
Consider the excerpt from Julie's explanation (see Table I) as another example. As can be seen the focus here is on the transfer of heat energy involved in the phenomenon. In addition to tracing the flow of energy, she also alludes to the principle of conservation of energy. Later in the interview she further clarifies her explanation by making the following argument: '...the egg originally inside before it's cooked is runny but as you transfer heat from the stove to the water, the water transfers heat to the egg which makes the liquidy bonds inside to be more solid bonds'. Textbook analysis reveals that the notion of heat as energy, the transfer of heat and conservation of energy are covered by all the three disciplines. Chemistry and physics covers additional details associated with these topics compared to biology. However, since the ideas involved in the excerpt shown in Table I are present in all three textbooks, we code the argument to be equally grounded in chemistry, physics and biology. In addition to energy and entropy, other ideas invoked by the students include mechanisms of heat transfer, structure of proteins, protein denaturation, bond breaking and formation, Gibbs free energy, ice-water phase transition etc.

Textbook analysis as described above becomes difficult if the student response lacks precision and certainty regarding the words used. For example, consider the following response to the egg phenomenon: 'So there is solidification and maybe some denaturing or hardening. Denaturing... I don't know if that's what's going on but that is this chemistry word there. Denaturing of enzymes. Or like, I don't know it's solidification of...'. The student in her struggle to come up with an explanation, is invoking a seemingly random bunch of vocabulary. Identification of knowledge elements is not possible and as such the response

[1] National Academy of Sciences, National Academy of Engineering, and Institute of Medicine of the National Academies, Facilitating interdisciplinary research (National Academies Press, Washington, DC, 2005).

[2] American Academy for the Advancement of Science, Vision and Change in Undergraduate Biology Education, (AAAS, Washington, DC, 2011).

[3] American Association of Physics Teacher, Conference on Introductory Physics for the Life Sciences Report, (AAPT, College Park, MD, 2015).

[4] National Research Council, A framework for K-12 science education: Practices, crosscutting concepts and core ideas (National Academies Press, Washington, DC, 2012)

[5] www.besocratic.com retrieved 8/29/2016. cannot be mapped to any discipline.

\section{OTHER CRITERIA FOR ASSESSING INTERDISCIPLINARITY: CONCLUDING REMARKS}

In this paper we discussed analyzing the extent to which students invoke different disciplines as one criterion for assessing interdisciplinarity. As we delve deeper into the data we will be exploring further criteria to develop a framework for assessing interdisciplinarity of student explanations of everyday phenomena. Other criteria in the literature include the strength of disciplinary grounding, degree of advancement achieved through integration of disciplines and critical awareness regarding clarity of purpose and limitations [9]. We maintain that assessment criteria should be true to where students are in their thinking, rather than ideals that are rarely achieved. As such appropriateness of the criteria present in the literature will be critically examined in the light of available data. An explanatory framework to characterize student reasoning of interdisciplinary phenomena, which is under development, is also expected to contribute valuable insights [10].

\section{ACKNOWLEDGEMENTS}

The authors wish to thank the CREATE for STEM Institute and the Physics Education Research Lab at Michigan State University. We are grateful to Angela Little for conducting the interviews. This work was funded by a LPF-CMP 2 Innovation grant.

[6] W. Bauer and G. D. Westfall, University Physics (McGraw Hill, New York, 2014)

[7] T. L. Brown, H. E. LeMay, B. E. Bursten, C. J. Murphy and P. M. Woodward, Chemistry-The Central Science (Pearson, Boston, 2012)

[8] S. Freeman, Biological Sciences (Pearson, San Francisco, 2011)

[9] V. B. Mansilla and E. D. Duraising, Targeted assessment of students interdisciplinary work: An empirically grounded framework proposed, Journal of Higher Education, Vol.78, No.2 (2007).

[10] E. E. Scott, V. D. Sawtelle, C. W. Anderson, K. K. Mashood, R. L. Matz and S. M. Underwood, Society for the Advancement of Biology Education Research (2016). 